
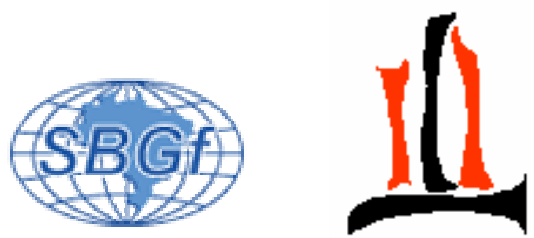

São Paulo 2004

\title{
Influência da silicificação na resistividade de rochas do Grupo Itararé - Bacia do Paraná
}

Campos, A. F. de ${ }^{1}$, Rigoti, A. ${ }^{2}$, Rostirolla, S. P. ${ }^{2}$, Appi, C. J. ${ }^{3}$, Kraft, R. P. ${ }^{1}$, Bartoszeck, M. K. ${ }^{1}{ }^{1}$ Bolsista CAPES - PósGraduação em Geologia/UFPR; ${ }^{2}$ Lab. de Análise de Bacias e Petrofísica-Degeol/UFPR; ${ }^{3}$ PETROBRAS/CENPES/PROFEX

Este texto foi preparado para a apresentação no I Simpósio Regional da Sociedade Brasileira de Geofísica, São Paulo, 26-28 de setembro de 2004. Seu conteúdo foi revisado pela Comissão Tecno-científica do I SR-SBGf mas não necessariamente representa a opinião da SBGf ou de seus associados. E proibida a reprodução total ou parcial deste material para propósitos comerciais sem prévia autorização da SBGf.

\section{Resumo}

Imageamento com eletrorresistividade foi utilizado para auxiliar na caracterização de análogos de reservatórios fraturados nos arenitos do Grupo Itararé, Bacia do Paraná. Este tipo de informação geofísica, correlacionada a outras fontes de dados como geológicos, sísmicos, estruturais e petrofísicos, permite uma visão mais refinada da subsuperfície. A área estudada está localizada próxima à cidade de Ponta Grossa (PR) e apresenta arenitos, folhelhos, diamictitos e conglomerados basais. O arranjo dipolo-dipolo foi usado objetivando um imageamento de resistividade. Os resultados mostram a associação da resposta geoelétrica com diversos aspectos geológicos como a geometria das camadas e o contato entre rochas diferentes. Outro aspecto a ressaltar é a provável correlação entre zonas fraturadas com elevados valores de resistividade por efeito da silicificação intensa.

\section{Introdução}

A distribuição de propriedades físicas, tais como resistividade elétrica, em associação a informações geológicas permite identificar e caracterizar feições geológicas e estruturais adicionais, importantes no estudo de detalhe de afloramentos similares a reservatórios fraturados como os enfocados neste trabalho.

Dentro do contexto do projeto Modelagem de Potenciais Reservatórios Fraturados em Superfície para Auxílio Exploratório em Subsuperfície (MODRES), a utilização do método de eletrorresistividade é uma ferramenta auxiliar na modelagem geométrica de reservatórios fraturados. Os dados de resistividade são integrados ao conjunto de dados que envolvem os sísmicos, geológicos, estruturais e petrofísicos para a modelagem do reservatório.

A área estudada é composta por arenitos e conglomerados basais, sobrepostos discordantemente a folhelhos marrom-chocolate, ritmitos e diamictitos.

Dentro da área de estudo, Rostirolla et al. (2000) individualizaram quatro subunidades faciológicas da Formação Campo Mourão, denominadas de Icm1 a Icm4, além de afloramentos da Formação Ponta Grossa, conforme pode ser visualizado na Figura 01. Através de levantamentos estruturais de detalhe, foi observado que os arenitos apresentam-se como camadas subhorizontais, basculadas a partir das falhas maiores, configurando um sistema de pequenos grabens e horsts.
Também foram reconhecidos elementos estruturais como bandas de deformação cimentadas por óxidos de ferro e/ou sílica, que afetam os arenitos de forma marcante e que provavelmente estão associados à evolução de uma zona de falha de caráter regional. $\mathrm{Na}$ área de estudo predomina um sistema de fraturas conjugadas, dispostas preferencialmente segundo as direções N10-20E e N60$80 \mathrm{~W}$.

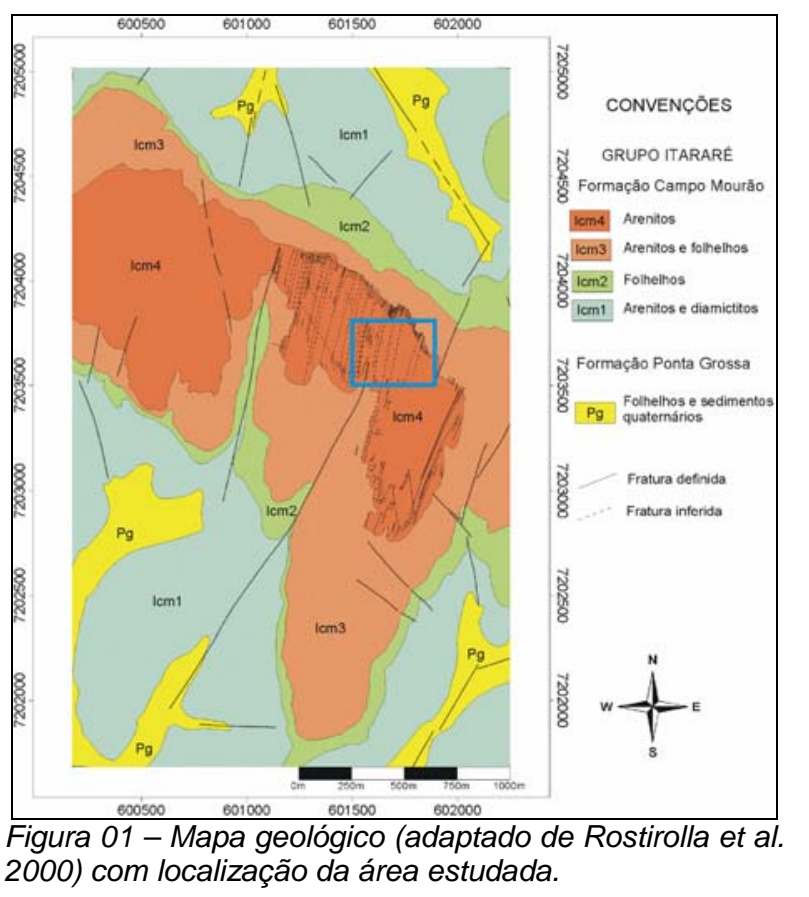

\section{O Método Geoelétrico}

A condução elétrica em sedimentos e rochas próximas a superfície é basicamente eletrolítica, ao longo dos poros interconectados. Esta condução é determinada pelos íons dissolvidos na água que preenche os poros e vazios.

O método de eletrorresistividade mede uma impedância, cuja interpretação permite avaliar a distribuição da resistividade elétrica e conseqüentemente, a geologia de subsuperfície. Uma corrente (I) é injetada no solo entre dois eletrodos (A e B) e a diferença de potencial $(\Delta V)$ é medida entre outros dois eletrodos (M e N). Na superfície da terra heterogênea e anisotrópica, mede-se uma resistividade aparente $\left(\rho_{\mathrm{a}}\right)$ calculada por:

$$
\rho a=\frac{\Delta V \times K}{I}
$$


onde $\mathrm{K}$ é um fator geométrico dependente do arranjo utilizado. A base teórica nas quais se fundamenta 0 método está disponível na literatura, como por exemplo Telford et al. (1990) e Keller \& Frischknecht (1977).

O levantamento de campo foi realizado sobre uma malha de treze linhas paralelas e distanciadas 10 metros, e uma oblíqua a estas, com aproximadamente 670 metros de comprimento.

Para o posicionamento das linhas no terreno, foi considerada a direção preferencial do sistema de fraturas, observado em fotografias aéreas na escala 1:10000 e em trabalhos de campo (Kraft et al., 2002), sendo dispostas perpendicularmente, ou seja, com direção aproximada SE-NW (Figura 02).

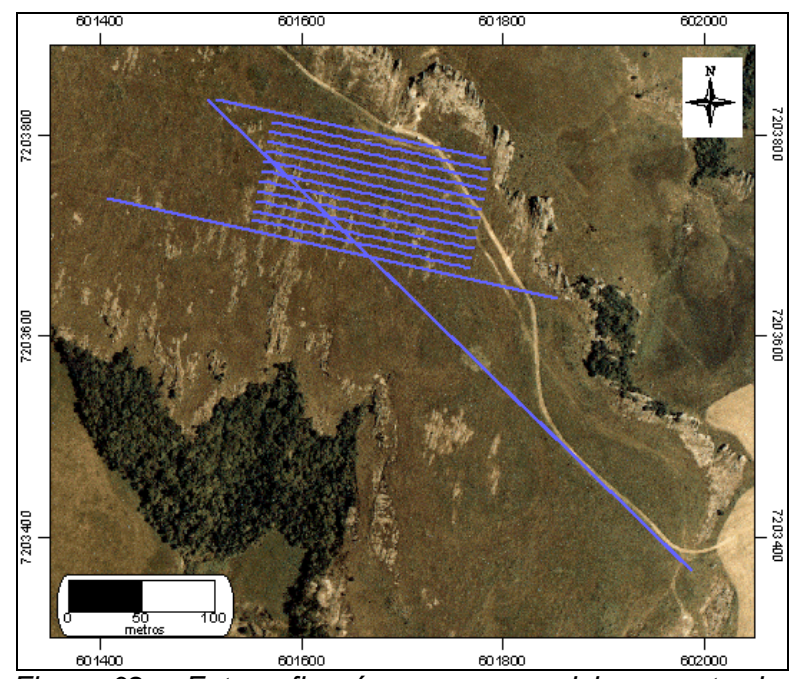

Figura 02 - Fotografia aérea com o posicionamento das linhas de levantamentos geoelétricos.

$\mathrm{O}$ arranjo utilizado no levantamento foi o dipolo-dipolo, com espaçamento entre os eletrodos de corrente $(A B)$ e de potencial $(\mathrm{MN})$ de $05,10,20,30$ e 40 metros. A escolha do arranjo dipolo-dipolo foi baseada no fato de que este arranjo é simétrico, de mais fácil interpretação e responde melhor a variações laterais de resistividade, como as esperadas para a área em estudo.

A fase de processamento iniciou-se com a plotagem dos dados na forma de pseudo-seções, para uma avaliação qualitativa da variação de resistividade e para um controle da qualidade dos dados. Após a verificação de que os dados de resistividade aparente não apresentavam problemas, foram preparados os arquivos para a inversão 2D. Na seqüência do processamento foi realizada uma análise estatística dos valores de resistividade e, a partir dela, foi criada uma escala de valores e legenda de cor única para todas as seções "reais". Este procedimento foi realizado para possibilitar uma correlação direta entre os valores de resistividade e as feições geológicas nas diversas seções geradas.

\section{Resultados}

Ao término da fase de processamento foram obtidos modelos de resistividade de todas as linhas levantadas. As Figuras 03 e 04 apresentam respectivamente, as seções modelo de resistividade referentes à primeira e segunda fase de levantamentos. Notadamente percebese os valores extremamente altos de resistividade (milhares de Ohm.m) distribuídos uniformemente em todos os modelos. Os valores elevados de resistividade refletem a baixa porosidade observada, $1 \%$ a $12 \%$, relatada em trabalhos de Bocardi et al. 2003. É importante ressaltar que as fraturas apresentam-se cimentadas por sílica e que esta situação reflete-se nos valores de resistividade observados. Em alguns casos, como na linha 04 , percebe-se que não ocorre uma alteração significativa na resistividade, apenas uma ligeira variação, não saindo da faixa de valores com alta resistividade. Desta forma pode-se identificar as zonas fraturadas pela análise de deslocamentos ou interferências nos valores de resistividade. Em contrapartida, na linha 03 é possível avaliar a influência das fraturas em porções mais superficiais, que podem estar mais abertas, permitindo desta forma a percolação por água e repercutindo em valores mais baixos de resistividade, embora ainda dentro de uma faixa considera alta para este caso.

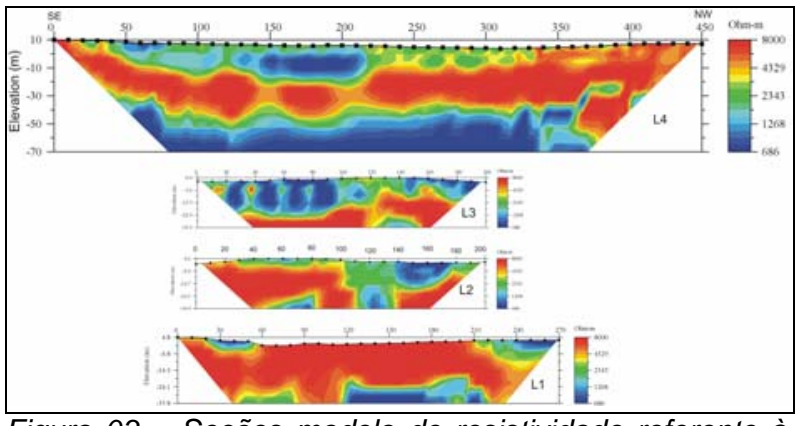

Figura 03 - Seções modelo de resistividade referente à primeira fase de levantamentos geoelétricos.

\section{Discussão e Conclusões}

A Figura 05 , referente à linha 04 , mostra que o modelo gerado pela inversão permitiu discriminar o contato entre os arenitos da subunidade $\mathrm{Icm} 4 \mathrm{com}$ os folhelhos da subunidade $\mathrm{Icm} 2$, sendo este contato representado por uma zona menos resistente iniciando-se há cerca de 50 metros de profundidade, sobreposta a uma camada mais resistente, associada aos arenitos da Icm4.

Outro aspecto a ressaltar nesta figura é a indicação de geometria lenticular da camada de arenitos da subunidade Icm4. Da mesma forma observa-se um deslocamento de resistividade, representando uma geometria semelhante a um graben. Como comentado anteriormente, também é possível identificar diversas fraturas em função da resposta geoelétrica, ou seja, através de feições como deslocamentos ou descontinuidades de resistividade.

A Figura 06 mostra a classificação dos modelos de resistividade em duas classes principais: uma zona de alta resistividade (cor cinza), com valores acima de 4000 Ohm.m, e uma zona de baixa resistividade (cor amarela).

Esta classificação foi utilizada para a interpretação das feições que foram associadas a fraturas. As fraturas em vermelho correspondem a feições relacionadas a zonas de alta resistividade, enquanto que as fraturas em azul foram traçadas analisando-se os baixos de resistividade no modelo. 
Campos, Rigoti, Rostirolla, Appi, Kraft e Bartoszeck

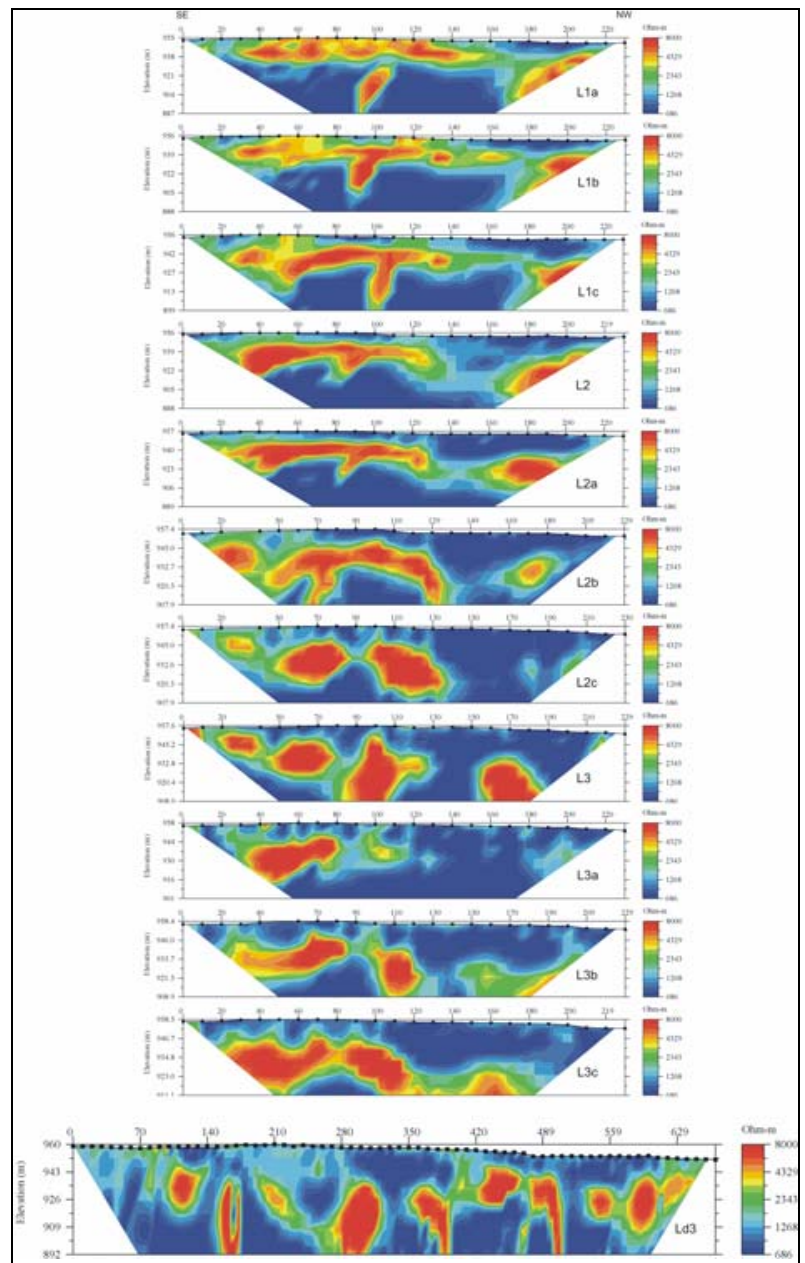

Figura 04 - Seções modelo de resistividade referente à segunda fase de levantamentos.

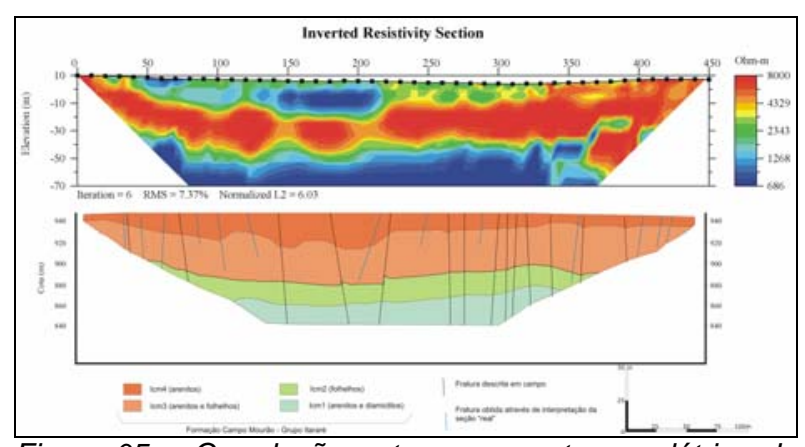

Figura 05 - Correlação entre a resposta geoelétrica do modelo de resistividade da linha 04 e informações geológicas e estruturais da área estudada.

Foi realizado o procedimento de transpor as fraturas interpretadas nos modelos para o mapa em planta, considerando-se que cada fratura no modelo corresponde a uma faixa em metros. Observa-se que houve uma associação entre os valores de resistividade e as fraturas mapeadas. Algumas fraturas, que ocorrem próximo à escarpa, apresentam-se mais descontínuas e com alta freqüência, possuem elevados valores de resistividade e são representadas por retângulos vermelhos. Esta situação pode ser explicada pelo fato destas fraturas encontrarem-se fechadas e silicificadas.
Em contrapartida, algumas fraturas apresentam-se mais contínuas e com menor freqüência, possuem baixos de resistividade associados que são representados por retângulos azuis. Podem ser explicadas pelo fato destas estarem mais abertas, facilitando percolação de água, e com porosidade mais alta associada.

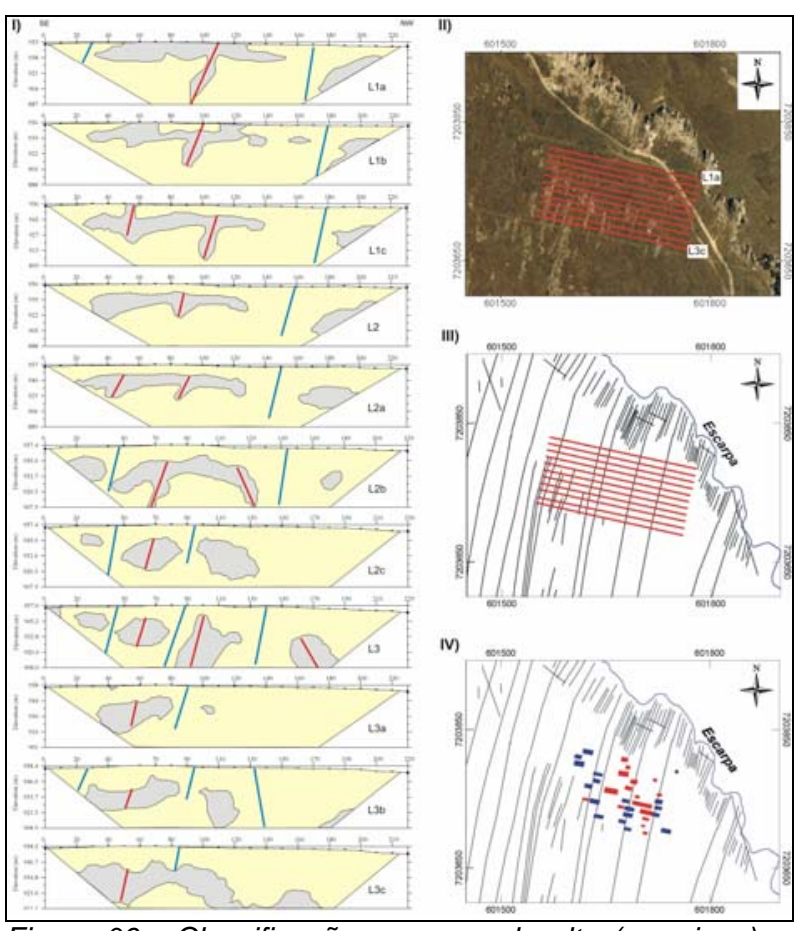

Figura 06 - Classificação em zona de alta (cor cinza) e baixa resistividade (cor amarela) e correlação entre as fraturas interpretadas a partir dos modelos de resistividade com fraturas mapeadas.

A Figura 07 apresenta os modelos de resistividade onde é possível acompanhar a continuidade de algumas fraturas com resposta resistente (linhas vermelhas) nas linhas $1 a, 1 b, 1 c, 2,2 a$ e $2 b$, através de uma faixa de influência (retângulo vermelho). Da mesma forma existe uma continuidade de algumas fraturas (linhas azuis) associadas a baixos valores de resistividade nas linhas $1 \mathrm{a}, 1 \mathrm{~b}, 1 \mathrm{c}, 2,2 \mathrm{a}, 2 \mathrm{~b}, 3$ e $3 \mathrm{~b}$, também nas linhas $2 \mathrm{~b}, 2 \mathrm{c}, 3$ e $3 b$ e também nas linhas $2 c, 3,3 a, 3 b$ e $3 c$, visualizadas através de uma faixa de influência representada por retângulos azuis.

Os resultados obtidos permitem correlacionar os valores de resistividade com feições geológicas, mais especificamente com a geometria das camadas e o contato entre litologias diferentes.

Altos de resistividade foram correlacionados a algumas fraturas mapeadas, possivelmente indicando que estas se encontram fechadas por efeito de intensa silicificação, enquanto que baixos de resistividade podem indicar fraturas mais abertas, com maior facilidade de percolação de água associada a uma porosidade maior.

De maneira geral foi observado que o condicionamento estrutural, representado pelas fraturas descritas, influenciam os valores de resistividade observados nos modelos. Isto pode ocorrer devido ao grau de silicificação e fechamento destas estruturas, repercutindo em zonas mais resistentes nos modelos observados. Da mesma forma foi possível identificar fraturas a partir de feições 
INFLUÊNCIA DA SILICIFICAÇÃO NA RESISTIVIDADE DE ROCHAS

geoelétricas que indicam deslocamentos ou descontinuidades de resistividade.

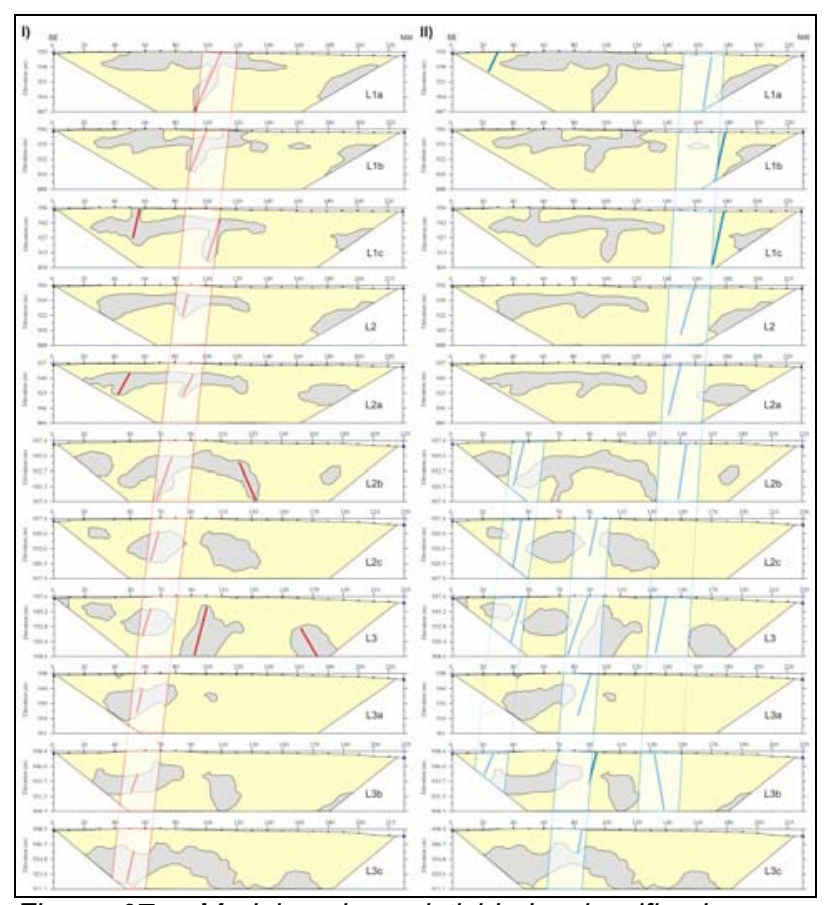

Figura 07 - Modelos de resistividade classificados em zonas de alta (cor cinza) e baixa (cor amarela) resistividade com indicação da área de possível influência de fraturas de alta (em vermelho) e de baixa resistividade (em azul).

A utilização de dados de resistividade, conjugados a outras informações geológicas permite identificar feições que auxiliam na caracterização de arenitos fraturados, que na área de estudo constituem uma situação geológica aflorante, similar a um reservatório fraturado.

Adicionalmente aos resultados descritos, a resistividade mostra valores elevados (milhares de Ohm.m), distribuídos de forma uniforme, mesmo nos níveis mais arenosos. Justamente estes níveis são os que poderiam hospedar fluidos e constituir reservatórios. A alta resistividade mostra contudo que ainda que a porosidade seja razoável, a permeabilidade da seção geológica analisada é muito baixa.

\section{Agradecimentos}

Os autores agradecem a CTPETRO/FINEP/PETROBRAS pelo financiamento, a UFPR pelo apoio institucional, ao CNPq pelas bolsas de pesquisa.

\section{Referências}

Bartoszeck, M.K.; Kraft, R.P.; Rostirolla, S.P.; Campos, A.F. de; Appi, C.J. 2003. Integração de dados geofísicos e geológicos como ferramenta auxiliar na modelagem de reservatórios em superfície. In: International Congress of The Brazilian Geophysical Society, 8, Rio de Janeiro, SBGf. CD.

Bocardi, L. B., Fernandes, L. A., Vesely, F. F., Rostirolla, S. P., Appi, C. J. 2003. Petrographic and diagenetic aspects of the Lapa and Vila Velha sandstones, Itararé Group, Paraná Basin, Brazil In: Latin American Congress of Sedimentology, 2003, Belém. anais.
Campos, A. F. de; Rigoti, A.; Rostirolla, S. P.; Appi, C. J.; Kraft, R. P.; Bartoszeck, M. K. 2003 Eletrorresistividade como apoio à caracterização de arenitos fraturados. In: International Congress of The Brazilian Geophysical Society, 8, Rio de Janeiro, SBGf. CD.

Keller, G.V. \& Frischknecht, F.C. 1977. Electrical methods in geophysical prospecting. Oxford: Pergamon Press, 1977, $517 \mathrm{p}$.

Kraft, R.P.; Rostirolla, S.P.; Bocardi, L.B.; Campos, A.F. de; Lonardelli, J.N. 2002. Análise petrofísica na caracterização de reservatórios de hidrocarbonetos um estudo nos arenitos "Lapa-Vila Velha" do Estado do Paraná. In: SBG, Congresso Brasileiro Geologia, 41, João Pessoa, Anais, 1:270.

Loke, M.H.; Barker R.D. 1996a. Rapid least-squares inversion of apparent resistivity pseudosections using a quasi-Newton method. Geophysical Prospecting, 44:131152.

Loke, M.H.; Barker R.D. 1996b. Practical techniques for 3D resistivity surveys and data inversion. Geophysical Prospecting, 44:499-523.

Lonardelli, J.N. do; Rigoti, A.; Rostirolla, S.P.; Appi, C.J.; Brunetta, R. 2003. Ensaios sísmicos de reflexão rasa em rochas fraturadas do Grupo Itararé. In: International Congress of The Brazilian Geophysical Society, 8, Rio de Janeiro, SBGf. CD.

Rostirolla, S.P; Appi, C.J.; Mancini, F.; Kraft, R.P.; Bartoszeck, M.K. 2000. Fraturas controladoras da migração de fluidos nos Arenitos Vila Velha, Grupo Itararé, Bacia do Paraná. Revista Brasileira de Geociências, 31:349-356.

Telford, W.M., Geldart, L.P., Sheriff, R.E. 1990. Applied geophysics. Second Edition. Cambridge: Cambridge University Press, 1990, 770 p. 